\section{SOI: 1.1/TAS DOI: 10.15863/TAS International Scientific Journal Theoretical \& Applied Science}

\author{
p-ISSN: 2308-4944 (print) e-ISSN: 2409-0085 (online) \\ Year: $2015 \quad$ Issue: $06 \quad$ Volume: 26
}

Published: $30.06 .2015 \quad$ http://T-Science.org

SECTION 4. Computer science, computer engineering and automation.
Tarandeep Singh

Master of Computer Application, Faculty GJIMT, Phase 2 Mohali,

Punjab Technical University, India tarandeepskhs@gmail.com

Parvinder Singh Sandhu

Ph.D, Professor in Computer Science \& Engineering department, Rayat \& Bahara Institute of Engineering and BioTechnology, India

Harbax Singh Bhatti Ph.D, Professor \& HOD (Applied Sciences) Baba Banda Singh Bahadur Engineering College, Fatehgarh Sahib, Punjab, India

\title{
RELIABILITY AND VALIDITY ANALYSIS ON MODEL FOR REDUCING THE WEIGHT AND LOAD ON PRODUCTION SERVER
}

Abstract: We describe Weibull reliability analysis on the model for weight and load reduction on the production server database for the mobile service transactions. We study report queries for which important load and weight of database. We study concept of load balancing of database queries and conceptually determine transaction replication to the reporting server database before purging in the conceptual model, which allow load balancing of the query and free space for the new transactions.

Key words: Reliability; Production Database; Reporting Database; 3P-Weibul, BX\% Life, MTTF, MTBF.

Language: English

Citation: Singh T, Sandhu PS, Bhatti HS (2015) RELIABILITY AND VALIDITY ANALYSIS ON MODEL FOR REDUCING THE WEIGHT AND LOAD ON PRODUCTION SERVER. ISJ Theoretical \& Applied Science 06 (26): 61-65.

Soi: http://s-o-i.org/1.1/TAS*06(26)14 Doi: crossef http://dx.doi.org/10.15863/TAS.2015.06.26.14

\section{Introduction}

The reliability of any system can be calculated as the probability to perform its purpose for a certain period of time in the specified environment $[1,2]$. Computation of reliability with simulation automated tools is popular, computation tools such as Weibull++ [3], which supports large base size and helps in controlling errors in computations. In this paper, we focus on the Reliability and load balancing of the Production server database and on managing and querying problem in the database. Different lifetime distribution representations have been used in Weibull++ to check which distribution perfectly fit for the data we have entered.

3P-Weibull is implemented on "model for reducing weight and load on production server database" [5] OLTP database is critical in reports extraction; OLTP database handles weight of transactions and load of reports. In this model we have demonstrated weight reduction of old transactions and load of reports on production server by making the reporting server capable enough to handle reports by replicating data on reporting server
(OLAP). As the data is available on reporting server we can now remove (Purge) some of the old data from production server database (OLTP), this task has been completed by partitioning the table and removal of partition and adding new partition, more space is free on the OLTP server for the new transactions and will increase the performance $[5,9,10,11,12,13]$. Our model used asynchronous mode of replication operation. The transactions first stored in the production server database and according to the server load the data will be replicated to the reporting server database [14]. We can adjust peak and non-peak hours of the database to increase and decrease the speed of the replication, this lead us to load reduction during peak hours. The reports can be extracted from the reporting server database without increasing any load on production server database [6].

\section{Objective}

This paper is designed to show the test of Reliability on the "Model for reducing weight and load on Production server database" [5] using 3PWeibull distribution method. We compute Probability of Failure, BX\% Life, Mean Life MTTF 
[2] and Failure Rate by using Weibull++ software's 3P-Weibull distribution method for the reliability.

\section{Minimum Reliability Test - Success Run Basic Properties}

In order to calculate reliability, tests are conducted with the sample data, now the question arises how high is the probability $P_{A}$ that a test sample falls during the test as per the literature [7, 8]:

$P_{A}=1-R_{t}^{n} \quad$ Where $R_{t}=$ Reliability at test time $t$ for test sample; $n=$ number of test specimen

Rearranging the formula:

$$
R_{t}=\left(1-P_{A}\right)^{\frac{1}{n}}
$$

The reliability for the test $\mathrm{t}$ "time" is calculated:

$$
R_{t}=e^{-\left(\frac{t}{T}\right)^{b}}
$$

Reliability $R_{a}$ applies to the defined service life $t_{a}$ :

$$
R_{a}=e^{-\left(\frac{t a}{T}\right) b}
$$

Equating the two relationships and defining $L_{v}=t / t_{a}$ results in:

$$
\frac{R_{t}}{R_{a}}=\frac{e^{-\left(\frac{t}{T}\right)^{b}}}{e^{-\left(\frac{t a}{T}\right)^{b}}} \rightarrow \frac{\ln \left(R_{t}\right)}{\ln \left(R_{a}\right)}=\frac{-\left(\frac{t}{T}\right)^{b}}{-\left(\frac{t a}{T}\right)^{b}}=L v^{b}
$$

As a result:

$$
\begin{gathered}
\ln \left(R_{t}\right)=\ln \left(R_{a}\right) L v^{b} \\
R_{t}=R_{a}^{L v^{b}}
\end{gathered}
$$

Together with the number of test specimens $R_{t}=\left(1-P_{A}\right)^{\frac{1}{n}}$ and equating results in:

$$
\begin{gathered}
R_{t}=R_{a}^{L_{v}^{b}}=\left(1-P_{A}\right)^{\frac{1}{n}} \\
R_{a}=\left(1-P_{A}\right)^{\frac{1}{n L_{v}^{b}}}
\end{gathered}
$$

The reliability $R_{a}$ taken as the "guaranteed minimum reliability" and applies the following:

$$
R_{\min }=\left(1-P_{A}\right)^{\frac{1}{n L_{v}^{b}}}
$$

\section{3-PWeibull and Sample Data from Model}

The reliability estimation on "model for reducing weight and load on production server database" $[8,5]$, 3P-Weibull reliability analysis with setting MLE (Maximum Likelihood), LRB (likelihood ratio) and K-M (Kaplan - Meier) [4].

In the Table 1 we have shown the results of 3PWeibull Parameters for the Reliability, Probability of Failure, BX\% Life, Mean Life and failure Rate.

3P-Weibull Parameters with Results.

Table 1

\begin{tabular}{|l|l|}
\hline 3P-Weibull Parameters & Results \\
\hline Reliability & $\mathrm{R}(\mathrm{t}=100)=0.905036$ \\
\hline Prob. of Failure & $\mathrm{Q}(\mathrm{t}=100)=0.094964$ \\
\hline BX\% Life & $\mathrm{B} 10 \%$ Life $=104.305977$ \\
\hline Mean Life & $\mathrm{MTTF}=735.254298$ \\
\hline Fail. Rate & Failure Rate $=0.001294$ \\
\hline
\end{tabular}


Impact Factor ISRA (India) $\quad=\mathbf{1 . 3 4 4}$

Impact Factor ISI (Dubai, UAE) $=\mathbf{0 . 8 2 9}$

based on International Citation Report (ICR)

Impact Factor GIF (Australia) $\quad \mathbf{0 . 3 5 6}$
Impact Factor JIF

$=\mathbf{1 . 5 0 0}$

Impact Factor SIS (USA)

$=0.912$

Impact Factor РИНЦ (Russia) $=0.179$

Impact Factor ESJI (KZ) $\quad=\mathbf{1 . 0 4 2}$

3P-Weibull standard folio.

Table 2

\begin{tabular}{|c|c|c|c|}
\hline Last Inspected (HR) & State F or S & Time to F or S (Hr) & Subset ID \\
\hline 1 & & 120 & 1 \\
\hline 1 & F & 120 & 1 \\
\hline 121 & S & 240 & 2 \\
\hline 121 & F & 240 & 2 \\
\hline 240 & S & 480 & 3 \\
\hline 240 & F & 480 & 4 \\
\hline 480 & S & 960 & 4 \\
\hline 480 & F & 960 & \\
\hline
\end{tabular}

Figure 1 gives an illustration of the Reliability vs. Time. With these number of samples collected from our model we have seen that the number of failures decrease by the increase of the usage of our model. 3P-Weibull standard folio shown in Table 2

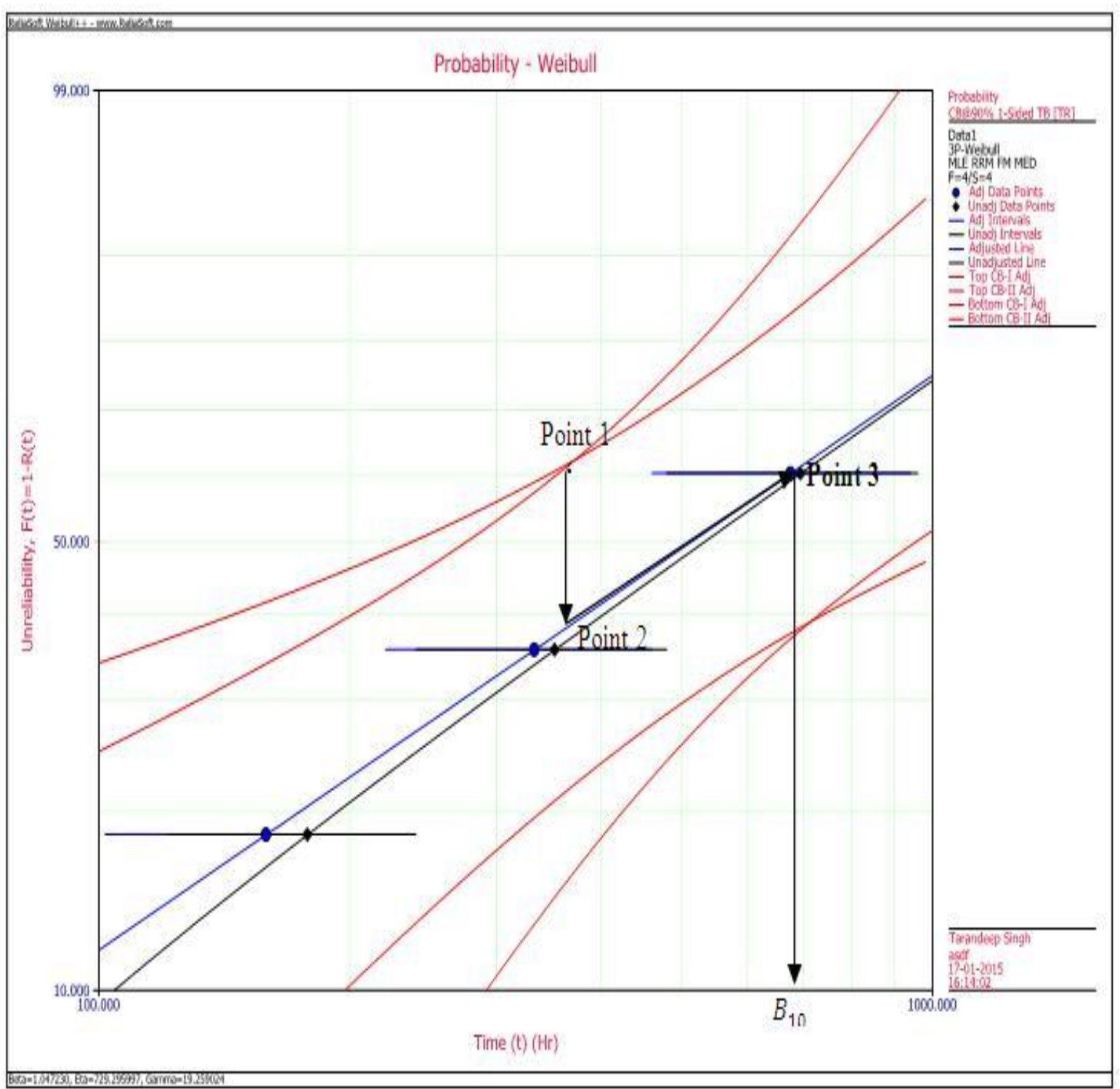

Figure 1 - Reliability vs. Time.

ISPC Education \& Innovation, 


\section{Determining minimum reliability without failures}

On examining the model in the Weibull plot the following representation is obtained $\left(P_{A}=0.85>\right.$ upper confidence bound $\left.90 \% t=1000 R_{\min }=90 \%\right)$ as shown in Table 1.

Minimum reliability for several tests with different running times used in the test as shown in Table 2, the running times are sorted in ascending order and the calculation started at the Subset ID 1 in the Table 2 .

\subsection{Determining $B X \%$ Life $\left(B_{1 o}\right)$ from minimum reliability}

For determining a minimum reliability $R_{\text {min }}$ from existing tests on our model Point (1) in the Figure 1 . The mean service life ratio $L_{v m}$, equivalent to the previous tests. The following formula is used $[7,8]$.

$$
\begin{aligned}
R_{\text {min }}=\left(1-P_{A}\right)^{\sum_{i=1}^{k} L v_{i}^{h} n_{i}-1}= & \\
& =\left(1-\left(1-P_{A}\right)^{\left(L v m^{b} n\right)^{-1}}\right.
\end{aligned}
$$

Rearranging the right side for $L_{v}$ results in :

$$
L_{v m}=\left(\frac{1}{n}\left(\frac{\ln (1-\mathrm{PA}}{\ln \left(\mathrm{R}_{\min }\right)}\right)\right)^{1 / \mathrm{b}}
$$

The reliability value on the Weibull curve with $P_{A}=50 \%$, the Point (2) in the Figure 1, compute as

$$
R_{P A=50 \%}=(1-0,5)^{1 /\left(L_{v m}^{b} n\right)}
$$

Now the Weibull curve is defined by specifying the slope $b$ and the Point (2) on the curve shown in Figure 1. After Rearranging the Weibull distribution for $T$ results in

$$
T=\frac{t}{(-\ln (l-H))^{1 / b}}=\frac{t_{\text {gefordert }}}{\left(-\ln \left(R_{P A=50 \%}\right)\right)^{1 / b}}
$$

$B_{10}$ Point (3) shown in Figure 1 calculated as

$$
B_{10}=T\left(\ln \left(\frac{1}{1-0,1}\right)\right)^{1 / b}
$$

\section{Mean Life MTTF}

Time is a common measure of life, Data points for life are also called "times-to-failure" and product life can be described in terms of time all the way through. The different types of life data provides different information about the life of the product, the analysis method can differ depending on the data type. Average time, the units in the collected data are expected to operate before failure. It is often referred to as mean time to failure (MTTF) or mean time between failures (MTBF) [2]. As it is made known in Table 1, that we have estimate MTTF $=735.254298$, on the "model for reducing weight and load on production server database" [5]. The data input in 3P-Weibull standard folio for working out of Mean life $[4,8]$ with Weibull++ software made known in Table 2.

The equation below is given for the $3 \mathrm{P}-\mathrm{Weibul}$ distribution.

$$
f(t)=\frac{\beta}{n}\left(\frac{\mathrm{t}-\gamma}{\mathrm{n}}\right)^{\beta-1} e^{-\left(\frac{\mathrm{t}-\gamma}{\mathrm{n}}\right)^{\beta}}
$$

$\eta$ defines where the bulk of the distribution lies.

$\beta$ defines the shape of the distribution

$\gamma$ defines the location of the distribution in time.

$t$ defines failure time.

During the analysis through Weibull++ software we have calculated the value Beta $=1.04722986475124$, $\mathrm{Eta}=729.295997246573$ and Gamma=19.2590244987316

\section{Failure Rate}

Failure rate is the calculation with which an engineered system, expressed in failures per hour. It is denoted by the letter $\lambda$ (lambda). It is closely related Mean Time between Failures (MTBF), more commonly expressed for high quality systems. Failure rate is generally time dependent, and the rate changes over time with the expected life cycle of a system [2]. The factors account for safety and maintenance practices in engineering and industry practices. A similar failure ratio used in the transport industries, such as railways and trucking is 'Mean Distance between Failure', which attempts to associate actual, loaded distances to like reliability needs and practices. Failure rates are vital factors in insurance, business, and guideline practices as well as essential to design of safe systems during a nationwide or global economy [7,8]. We have calculated Failure Rate $=0.001294$ on "Model for reducing weight and load on production server database" [5], with Weibull++ software [4], as shown in Table 1.

\section{Prob. of Failure}

If the Weibull shape factor is greater than one the analysis is indicating that rising hazard conditions apply. The probability of failure is therefore rising with time; the higher the $\beta$ value, the greater is the rate of increase. This is often called the 'wear-out' phase, although again this term can be ambiguous. The time reliance of failures now permits 
levelheaded consideration of planned replacement providing the total cost of a failure replacement is greater than the total cost of a planned replacement. The interval for such replacements should be optimized and there is at least one general technique which will do this directly from the Weibull parameters [7].

The outcome of the Probability of failure $\mathrm{Q}(\mathrm{t}=100)=0.094964$ on "Model for reducing weight and load on production server database" [5], shown in Table 1.

\section{Conclusion and future work}

In this paper we discussed the reliability estimation of "model for reducing weight and load on production server database" [5] based on 3-P Weibull distribution method. We have calculated
Failure Rate, Probability of Failure, minimum reliability without any failures, $\mathrm{BX} \%$ Life and mean time to failure (MTTF) on the data which have been recorded during the hypothesis working of the model. 3P-Weibull with analysis setting MLE (Maximum Likelihood), LRB (likelihood ratio) and K-M (Kaplan - Meier). The Weibull++ simulator is used for the reliability estimation. Further work can be done in this area using other methods such as $2 \mathrm{P}$ Exponential, 1P-Exponential, G-Gama.

\section{Acknowledgement}

This research is supported by the Research Center (RIC PTU), Punjab Technical University, Jalandhar - Kapurthala Highway, Kapurthala, Punjab (India).

\section{References:}

1. Waseem Ahmed, YongWeiWu (2013) "A survey on reliability in distributed systems", Journal of Computer and System Sciences, vol. 79, pp. 1243-1255.

2. NaeemAbbasi, OsmanHasan, SofièneTahar (2013) “ An approach for lifetime reliability analysis using theorem proving", Journal of Computer and System Sciences, vol. 80, pp. 323-345.

3. Yi Lua, Qiaomin Xie, Gabriel Kliot, Alan Geller, James R. Larus, Albert Greenberg (2011) "Join-Idle-Queue: A novel load balancing algorithm for dynamically scalable web services", Performance Evaluation, vol 68, pp. 1056-1071.

4. (2015) Weibull Distribution Examples. Available:

http://reliawiki.org/index.php/Weibull_Distribut ion_Examples (Accessed: 15.06.2015).

5. Tarandeep Singh, Parvinder S. Sandhu, Harbax Singh Bhatti (2014) "Model for Reducing Weight and Load on Production Server Database",Planetary Scientific Research Center Proceeding Johannesburg (South Africa), ISBN 978-93-82242-71-0, April 15-16, 2014.

6. Tarandeep Singh, Parvinder S. Sandhu, Harbax Singh Bhatti (2013) Replication of Data in Database Systems for Backup and Failover- An Overview, International Journal of Computer and Communication Engineering, Vol. 2, No. 4, pp. 535-538, DOI: 10.7763/IJCCE.2013.V2.243

7. Dr. Robert. Abernethy (2006) The New Weibull Handbook Fifth Edition, Reliability and Statistical Analysis for Predicting Life, Safety, Supportability, Risk, Cost and Warranty Claims, November 1, 2006.
8. J-P. Clech, D. M. Noctor, J. C. Manock, G. W. Lynott and F.E. Bader (1994) AT\&T Bell Laboratories, "Surface Mount Assembly Failure Statistics And Failure-Free Times", 44th ECTC, Washington, D.C., May 1-4, 1994, pp. 487-497.

9. Dany Maslowski, Jef Wijsen (2013) "A dichotomy in the complexity of counting database repairs", Journal of Computer and System Sciences Volume 79, Issue 6, pp. 958983.

10. Mahabadi, S.M. Zahedi, A. Khonsari (2013) "Reliable energy-aware application mapping and voltage-frequency island partitioning for GALS-based NoC", Journal of Computer and System Sciences vol. 79, pp. 457-474.

11. Bart Kuijpers, Walied Othman (2010) "Trajectory Databases: Data Models, Uncertainty And Complete Query Languages", Journal of Computer and System Sciences vol. 76, pp. 538-560.

12. Riccardo Rosati (2011) "On The Finite Controllability Of Conjunctive Query Answering In Databases Under Open-World Assumption", Journal of Computer and System Sciences vol. 77, pp. 572-594.

13. Karen Works, Elke A. Rundensteiner, Emmanuel Agu (2013) "Optimizing adaptive multi-route query processing via timepartitioned indices" Journal of Computer and System Sciences vol.79, pp. 330-348.

14. Paolo Romano, Roberto Palmieri, Francesco Quaglia, Nuno Carvalho, LuisRod rigues (2014) "On speculative replication of transactional systems", Journal of Computer and System Sciences vol. 80, pp.257-276. 